\begin{abstract}
HEAT TRANSFER ASIAN RESEARCH
EDITOR-IN-CHIEF: PROFESSOR William. M. Worek

Frank H. Dotterweich College of Engineering, Texas A\&M University-Kingsville, Kingsville, Texas, USA.
\end{abstract}

PUBLISHER- WILEY USA; ONLINE ISSN: 1523-1496

ACCEPTED OCTOBER $5^{\text {TH }} 2018$

\title{
APPLICATION OF DIFFERENTIAL TRANSFORM METHOD TO UNSTEADY FREE CONVECTIVE HEAT TRANSFER OF A COUPLE STRESS FLUID OVER A STRETCHING SHEET
}

\author{
Mahesh Kumar ${ }^{1}$, G. Janardhana Reddy ${ }^{1, *}$, N. Naresh Kumar ${ }^{2}$ and O. Anwar Bég ${ }^{3}$ \\ ${ }^{1}$ Department of Mathematics, Central University of Karnataka, Kalaburagi, India. \\ ${ }^{2}$ School of Humanities \& Sciences, SASTRA Deemed University, Thanjavur, Tamil Nadu, India \\ ${ }^{3}$ Fluid Mechanics, Aeronautical and Mechanical Engineering Department, School of Computing, \\ Science and Engineering, University of Salford, Manchester M54WT, UK
}

*Corresponding author-Email: gjr@cuk.ac.in

\section{ABSTRACT}

In the present article, the transient rheological boundary layer flow over a stretching sheet with heat transfer is investigated, a topic of relevance to non-Newtonian thermal materials processing. Stokes couple stress model is deployed to simulate non-Newtonian characteristics. Similarity transformations are utilized to convert the governing partial differential equations into nonlinear ordinary differential equations with appropriate wall and free stream boundary conditions. The non-dimensional boundary value problem emerging is shown to be controlled by a number of key thermophysical and rheological parameters, namely the rheological couple stress parameter $(\beta)$, unsteadiness parameter $(A)$, Prandtl number $(P r)$, buoyancy parameter $(\lambda)$. The semi-analytical Differential Transform Method (DTM) is used to solve the reduced nonlinear coupled ordinary differential-boundary value problem. A numerical solution is also obtained via the MATLAB builtin solver 'bvp4c' to validate the results. Further validation with published results from the literature is included. Fluid velocity is enhanced with increasing couple stress parameter whereas it is decreased with unsteadiness parameter. Temperature is elevated with couple stress parameter whereas it is initially reduced with unsteadiness parameter. The flow is accelerated with increasing positive buoyancy parameter (for heating of the fluid) whereas it is decelerated with increasing negative buoyancy parameter (cooling of the fluid). Temperature and thermal boundary layer thickness are boosted with increasing positive values of buoyancy parameter. Increasing Prandtl number decelerates the flow, reduces temperatures, increases momentum boundary layer thickness and decreases thermal boundary layer thickness. Excellent accuracy is achieved with the DTM approach.

KEYWORDS: Couple stress fluid; DTM; Stretching sheet; Heat transfer; Boundary layers. 


\section{INTRODUCTION}

Convective heat transfer is a rich area of engineering science and has been extensively studied both experimentally and theoretically for many decades. Convective heat transfer occurs whenever a fluid approaches in contact with a surface whose temperature is different from its own. Convective heat transfer in general refers to heat movement in fluids (liquids and gases). It is a process which takes place in fluid flow over a hot or cold surface whereby the fluid flow acts as a carrier for energy. As such convective heat transfer is fundamental to many diverse applications in technology, medicine and nature. These include flows in automobile radiators, domestic and industrial heating or cooling devices (heaters, condensers and boilers), electronic component cooling, crystal growth, direct absorption solar collectors, geophysical plumes, blood flows, diffusion flames in fire dynamics etc. When buoyancy forces are dominant natural (free) convection occurs and in the absence of buoyancy forces forced convection prevails.

From an industrial viewpoint, the analysis of the flow driven by a stretching surface is of significant practical interest. It features in glass crystal growing, fibre drawing, continuous casting, plastic extrusion, drawing of copper wires, coating of components etc. The viscous fluid flow problem for a continuous moving sheet was first examined in the context of chemical processing by Sakiadis [1]. Crane [2] extended the Sakiadis model work for two-dimensional stretching surface flow and presented an exact solution. Subsequently many researchers have further extended the Sakiadis and Crane models to present similarity solutions for mixed convection [3], stagnation-point convection [4], heat generation/absorption effects on convection flows [5] and stagnation flows in permeable media [6]. On the other hand, in many stretching flows, time-dependent behaviour arises. This characterizes for example the flow from an impulsive stretching of the sheet or the flow induced by a step change of the temperature. When the surface is stretched suddenly with a definite velocity, the flow is developed instantaneously. Interesting simulations of transport phenomena associated with the impulsive stretching of a sheet have been reported in [7] (for vertical surfaces), [8] (for liquid film dynamics), [9] (stretching with wall cooling), [10] (multiple methods of solution given including perturbation for small times, numerical integration, and asymptotic analysis for large times), [11] (thermo-convection over a wide range of Prandtl numbers), [12] (stretching stagnation flow with 
stretching velocity proportional to the distance from the stagnation point) and [13] (thermo-solutal convection).

The above studies were all confined to Newtonian fluids i.e. they ignored rheological (nonNewtonian) characteristics which are important in for example polymer processing, coating protection, extrusion of plastics, glass blowing, etc., An extensive range of non-Newtonian models have been developed which accurately mimic many complex features of real industrial fluids including relaxation, retardation, visco-plasticity, viscoelasticity, spurt, normal stress differences etc. Rajagopal et al. [14] examined the flow of a second order Reiner-Rivlin viscoelastic fluid over a stretching sheet. Bég et al. [15] used a network simulation method to compute the stretching flow of a magnetic Walters-B viscoelastic fluid with time-dependent wall suction in Darcy-Forchheimer porous media. Chen et al. [16] also used the Walters-B ("short memory") viscoelastic model to study the non-Newtonian thermal convection flow from a stretching plate, noting that temperature decreases with lower values of the viscoelastic parameter. Abel et al. [17] scrutinized the power law fluid flow over a stretching sheet. Sahoo [18] examined the flow of third grade fluid from stretching sheet. Recently the flow of non-Newtonian Williamson fluid due to a stretching surface was analysed by Hamid et al. [19]. The rheological models utilized in these studies however neglected an important characteristic of many technological fluids, namely micro-structure. Amongst non-Newtonian fluids, the Stokes couple stress fluid is the simplest generalization of the classical theory of fluids which allows for polar effects such as the presence of couple stresses and body couples [20]. Lubricant oils with long chain additives, blood, polymeric suspensions, slurries, colloids and other industrial liquids constitute some common examples of couple stress fluid. The couple stress fluid model extends the conventional Cauchy stress featured in Navier-Stokes (Newtonian) models and invokes length dependent effects which are associated with particles suspended in industrial fluids (polymers). These give rise to couple stresses which can exert a non-trivial influence on shear (and other) characteristics of such liquids which in turn can influence heat transfer characteristics. Couple stress or "polar" fluid models therefore provide a more elegant formulation than the classical non-polar models. They also have the advantage that although they lead to boundary value problems with higher order than the Navier-Stokes, the supplementary terms are linear. This feature has stimulated considerable interest among engineers and mathematicians. Many complex industrial systems have been analysed using the couple stress model. Bég et al. [21] investigated magnetic centrifugal blood processing devices using Stokes couple stress model noting that couple stresses have a profound influence on wall shear stress, swirl and heat transfer characteristics. Ramana Murthy et al. [22] studied multi-mode heat transfer in channel flow of two immiscible couple stress fluids using second law thermodynamic 
optimization. Tripathi et al. [23] derived analytical solutions for electrokinetic peristaltic pumping of couple stress physiological fluids under an axial electrical field in microfluidic systems. The couple stress fluid theory has also been applied to a number of stretching sheet flow problems. Khan et al. [24] studied heat transfer in couple stress fluid from a nonlinearly stretching sheet for prescribed surface temperature and prescribed heat flux with a shooting method. Hayat et al. [25] investigated chemically-reacting couple stress stretching sheet flow. Hayat et al. [26] presented homotopy solutions for melting in stagnation flow of couple stress fluids, observing that temperature and surface heat transfer are elevated with couple stress effect whereas the flow is decelerated. Mahabaleshwar et al. [27] derived power series and Kummer confluent hyper-geometric function solutions for magnetohydrodynamic couple stress flow and convection from a linearly stretching sheet under a variety of different boundary conditions. These studies all confirmed the significant impact that couple stresses exert on heat and momentum transfer characteristics.

Most phenomena in fluid mechanics are essentially nonlinear in nature and are described by nonlinear partial or ordinary differential equations. Purely analytical methods cannot solve these nonlinear equations exactly. Other methods which are semi-analytical/numerical in nature therefore must be implemented and some popular methods are the Homotopy Analysis Method (HAM), Adomian Decomposition Method (ADM), Homotopy Perturbation Method (HPM), Optimal Homotopy Asymptotic Method (OHAM) and the Differential Transformation Method (DTM). These methods are frequently used owing to their high accuracy and simplicity in yielding solutions. In the present article we employ the Differential Transformation Method (DTM) [28] which is a robust semi-exact method and is not based on the existence of small or large parameters. A close inspection of the previous literature study indicates that very limited studies have been reported on the application of DTM to nonlinear time-dependent flows of couple stress fluids from a stretching sheet. Hence, the current study aims to apply DTM to the boundary layer flow of couple stress fluid with heat transfer from a stretching sheet. Extensive details of the mathematical model, transformation, boundary conditions, DTM solution and graphical results for various emerging parameters are included. A rigorous discourse on the physical implications of the computed solutions is provided with validation of solutions (where possible) with previous studies and a shooting numerical method. The present work aims to provide a deeper insight into the thermofluid characteristics of rheological materials processing and has not been reported thus far in the technical literature. 


\section{MATHEMATICAL FORMULATION}

Time-dependent, two-dimensional, laminar, incompressible couple stress fluid flow and thermal convection heat transfer from a stretching sheet is considered as depicted in Fig. 1. At the outset i.e., $t=0$, the sheet is impulsively stretched with velocity $U_{w}(x, t)$ along the $x$-axis, keeping the origin fixed in the fluid which has a free stream temperature $T_{\infty}$. The temperature of the sheet $T_{w}(x, t)$ is assumed to be a linear function of the $x$-coordinate. The fixed Cartesian coordinate scheme has its origin positioned at the foremost verge of the sheet (slit where the sheet emerges) with the positive $x$-axis extending along the upward direction of sheet and the $y$-axis orientated normal to the surface of the sheet. Under these assumptions (with the boundary layer and Boussinesq approximations), the governing equations for the mass, momentum and energy conservation in mixed convective flow and heat transfer of the couple stress fluid, obtained by adding couple stress and heat effects to the models of [1], [2] are:

$$
\begin{gathered}
\frac{\partial u}{\partial x}+\frac{\partial v}{\partial y}=0 \\
\rho\left(\frac{\partial u}{\partial t}+u \frac{\partial u}{\partial x}+v \frac{\partial u}{\partial y}\right)=g \beta_{T}\left(T-T_{\infty}\right)+\mu \frac{\partial^{2} u}{\partial y^{2}}-\eta_{1} \frac{\partial^{4} u}{\partial y^{4}} \\
\frac{\partial T}{\partial t}+u \frac{\partial T}{\partial x}+v \frac{\partial T}{\partial y}=\alpha \frac{\partial^{2} T}{\partial y^{2}}
\end{gathered}
$$

The associated boundary conditions on the stretching sheet (wall) and in the free stream (edge of the boundary layer) are given by:

$$
\begin{gathered}
T=T_{w}, v=0, u=U_{w} \quad \text { at } y=0 \\
T \rightarrow T_{\infty}, u \rightarrow 0 \quad \text { as } y \rightarrow \infty \\
\frac{\partial^{2} u}{\partial y^{2}}=0 \text { at } y=0, u \rightarrow 0 \text { and } \frac{\partial u}{\partial y} \rightarrow 0 \text { as } y \rightarrow \infty
\end{gathered}
$$

We assume that the stretching velocity $U_{w}(x, t)$ and the surface temperature $T_{w}(x, t)$ are of the form

$$
U_{w}(x, t)=\frac{a x}{(1-c t)} \text { and } T_{w}(x, t)=T_{\infty}+\frac{b x}{(1-c t)^{2}}
$$

where $a$ and $c$ are constants (with $a>0$ and $c \leq 0$, where $c t<1$ ), and both have dimension of frequency (inverse of time), while $b$ is a constant and has dimension temperature/length, with $b>0$ 
and $b<0$ relate to the assisting and opposing flows, respectively. Furthermore $b=0$ corresponds to forced convection limit (absence of buoyancy force). It is noteworthy that at $t=0$ (initial motion), these particular forms of $U_{w}(x, t)$ and $T_{w}(x, t)$ have been preferred in order to extract a new similarity transformation, which converts the governing partial differential Eqns. (1) - (3) into a set of ordinary differential equations. Progressing with the analysis, let us introduce the following dimensionless functions $f$ and $\theta$, and similarity variable $\eta$ as follows $[7,8]$ :

$$
\eta=\sqrt{\frac{a}{v(1-c t)}} y, \psi=\sqrt{\frac{a v}{(1-c t)}} x f(\eta), \theta(\eta)=\frac{T-T_{\infty}}{T_{w}-T_{\infty}},
$$

Here $\psi(x, y, t)$ is the stream function defined as $(u, v)=\left(\frac{\partial \psi}{\partial y},-\frac{\partial \psi}{\partial x}\right)$ which identically satisfies the continuity (mass conservation) equation (1). Substituting Eqn. (6) into (2) and (3) we obtain:

$$
\begin{gathered}
\beta f^{v}-f^{\prime \prime \prime}+f^{\prime 2}-f f^{\prime \prime}+A\left(\frac{1}{2} \eta f^{\prime \prime}+f^{\prime}\right)-\lambda \theta=0 \\
\frac{1}{P r} \theta^{\prime \prime}+f \theta^{\prime}-\theta f^{\prime}-A\left(\frac{1}{2} \eta \theta^{\prime}+2 \theta\right)=0,
\end{gathered}
$$

Here prime denotes differentiation with respect to $\eta, \beta=a \eta_{1} / v^{2} \rho(1-c t)$ is the couple stress (nonNewtonian) parameter, $A=c / a$ is the unsteadiness parameter, $\operatorname{Pr}=v / \alpha$ is the Prandtl number. Also, $\lambda$ is the buoyancy parameter which is defined as $\lambda=G r / R e^{2}$ where $G r=g \beta_{T}\left(T_{w}-T_{\infty}\right) x^{3} /$ $v^{2}$ denotes local Grashof number and $R e=U_{w} x / v$ denotes the local Reynolds number. Here, $\lambda$ is dimensionless constant with $\lambda<0$ and $\lambda>0$ associated respectively with opposing flow and assisting flow cases, whereas $\lambda=0$ relates to the forced convection flow situation (vanishing buoyancy force). The boundary conditions transform to:

$$
\begin{array}{cc}
f(0)=0, f^{\prime}(0)=1, f^{\prime \prime \prime}(0)=0, \theta(0)=1 & \text { at } \eta=0 \\
f^{\prime}(\eta) \rightarrow 0, f^{\prime \prime}(\eta) \rightarrow 0, \theta(\eta) \rightarrow 0 & \text { as } \eta \rightarrow \infty
\end{array}
$$

The local skin-friction coefficient $C_{f}$ and local Nusselt number $N u$ are important wall gradient characteristics associated with thermal rheological materials processing. They are defined as follows:

The surface shear stress $\tau_{\mathrm{w}}$ is given by [28-32]:

$$
\tau_{w}=\left(\mu\left(\frac{\partial u}{\partial y}\right)-\eta\left(\frac{\partial^{3} u}{\partial y^{3}}\right)\right)_{y=0}
$$

Using similarity transformation from Eq. (6), the shear stress $\tau_{\mathrm{w}}$ becomes 


$$
\tau_{w}=v \sqrt{\frac{a}{(1-c t)}} \frac{a x}{(1-c t)}\left(f^{\prime \prime}(0)-\frac{a \eta_{1}}{\rho v^{2}(1-c t)} f^{\prime \prime \prime \prime}(0)\right)
$$

The local skin-friction coefficient $C_{f}$ is given by

$$
\begin{aligned}
& C_{f}=\frac{\tau_{w}}{\rho U_{w}{ }^{2} / 2}, \quad \frac{1}{2} C_{f} R e^{1 / 2}=f^{\prime \prime}(0)-\beta f^{\prime \prime \prime \prime}(0) \\
& N u=\frac{q_{w}}{T_{w}-T_{\infty}}\left(\frac{x}{k}\right), q_{w}=-k\left(\frac{\partial T}{\partial y}\right)_{y=0} \text { then } N u / R e^{1 / 2}=-\theta^{\prime}(0)
\end{aligned}
$$

\section{SOLUTION OF THE PROBLEM}

The nonlinear non-dimensional ordinary differential boundary value problem defined by Eqns. (7)(9) is solved with DTM. The concept of DTM was first suggested by Zhou in 1986 [33] and it was applied to elucidate linear and non-linear initial value problems in electric circuit analysis. DTM is based on a Taylor-series method and constructs an analytical solution in the form of a polynomial. The main benefit of this method is that it can be applied directly to nonlinear differential equations without requiring discretization, linearization, and therefore, it is not affected by errors associated with discretization. This method has been successfully implemented in numerous multi-physical mechanics, fluid dynamics and heat transfer problems in recent years. These include nonlinear thermal conduction [34], hypersonic heating in boundary layers [35], haemotological filtration dynamics [36], swirl vortex nuclear magnetic propulsion thermodynamics [37], digestive transport modelling [38], thermo-solutal convection in porous media [39], nanoscale fluid dynamics [40], micropolar fluid flows [41,42], chemically-reacting flows in permeable materials [43] and biomagnetic entropy generation in hemodynamics [44]. DTM has been shown to be very efficient in these studies. Although convergence may be accelerated with modifications of this method e.g. Padé approximations, it is not necessary. The method and its application to the current problem are now described. The differential transformation of the $k$ th derivative of function $f(\eta)$ is defined as follows:

$$
F(k)=\frac{1}{k !}\left[\frac{d^{k} f(\eta)}{d \eta^{k}}\right]_{\eta=\eta_{0}}
$$

where $f(\eta)$ is the original function and $F(k)$ is the transformed function. The inverse differential transformation is as follows:

$$
f(\eta)=\sum_{k=0}^{\infty} F(k)\left(\eta-\eta_{0}\right)^{k}
$$

In real applications, the function $f(\eta)$ is expressed by a finite series and Eqn. (12) can be written as: 


$$
f(\eta)=\sum_{k=0}^{m} F(k)\left(\eta-\eta_{0}\right)^{k} .
$$

Eqn. (13) implies that $\sum_{k=m+1}^{\infty} F(k)\left(\eta-\eta_{0}\right)^{k}$ is negligibly small. In fact $m$ is dictated by the convergence in this study. Some of the properties of DTM are shown in Table 1. These properties are derived from Eqns. (12) and (13). Applying DTM to the non-linear governing conservation Eqns. (7) and (8) along with boundary conditions (9) we obtain following recurrence relations:

$$
\begin{gathered}
\beta(k+1)(k+2)(k+3)(k+4)(k+5) F(k+5)-(k+1)(k+2)(k+3) F(k+3)+ \\
\sum_{r=0}^{k}(F[k-r](k+1)(k+2) F(k+2)) \quad-\sum_{r=0}^{k}((k-r+1) F[k-r+1](k+1) F(k+1))+ \\
A\left(\frac{1}{2} \sum_{r=0}^{k}(\delta(k-r+1)(k+1)(k+2) F(k+2))+(k+1) F(k+1)\right)-\lambda T[k]=0 \\
\frac{1}{P r}(k+1)(k+2) T(k+2)-A\left(\frac{1}{2} \sum_{r=0}^{k}(\delta(k-r+1)(k+1) T(k+2))+2 T(k)\right) \\
+\sum_{r=0}^{k}(F[k-r+1](k+1) T(k+1))-\sum_{r=0}^{k}(T[k-r+1](k+1) F(k+1))=0 \\
F[0]=0, F[1]=1, F[2]=\frac{n_{1}}{2}, F[3]=0, F[4]=\frac{n_{2}}{24} \\
T[0]=0, T[1]=n_{3}
\end{gathered}
$$

where $F(k), T[k]$ are the differential transform of $f(\eta), \theta(\eta)$ and $n_{1}, n_{2}, n_{3}$ are constants which can be obtained through boundary conditions i.e. Eqn. (9).

Table 1: Properties of DTM [33]

\begin{tabular}{|l|c|}
\hline Original function & Transformed function \\
\hline$f(\eta)=g(\eta) \pm h(\eta)$ & $F(k)=G(k) \pm H(k)$ \\
\hline$f(\eta)=c g(\eta)$ & $F(k)=c G(k)$ \\
\hline$f(\eta)=\frac{d^{n} g(\eta)}{d \eta^{n}}$ & $F(k)=\frac{(k+n) !}{k !} G(k+n)$ \\
\hline$f(\eta)=g(\eta) h(\eta)$ & $F(k)=\sum_{r=0}^{k} G(r) H(k-r)$ \\
$f(\eta)=\eta^{n}$ & $F(k)=\delta(k-n)= \begin{cases}1, \text { if } k=n \\
0, \text { if } k \neq n\end{cases}$ \\
\hline
\end{tabular}

Using the transformed boundary conditions (16a) and (16b) we obtained following recurrence relations: 


$$
\begin{aligned}
& F[5]=\frac{\lambda-1-\mathrm{A}}{120 \beta}, \\
& F[6]=\frac{\lambda n_{3}+n_{2}-n_{1}(1+\mathrm{A})}{720 \beta}, \\
& F[7]=\left(\frac{\lambda-(1+\mathrm{A})}{2 \beta}+\frac{1}{2} \operatorname{Pr} \lambda(1+2 \mathrm{~A})-\frac{n_{1}^{2}}{2}\right) / 2520 \beta, \\
& F[8]=\left(\frac{\lambda n_{3}+n_{2}-n_{1}(1+\mathrm{A})}{6 \beta}+\frac{1}{6} \operatorname{Pr} \lambda\left(n_{1}+2 \mathrm{~A} n_{3}\right)+\frac{n_{2}}{6}-\frac{\mathrm{A} n_{2}}{6}\right) / 6720 \beta, \\
& F[9]=\left(\begin{array}{c}
\frac{\frac{\lambda-(1+A)}{2 \beta}+\frac{1}{2} \operatorname{Pr} \lambda(1+2 \mathrm{~A})-\frac{n_{1}{ }^{2}}{2}}{12 \beta}-\frac{\mathrm{A}(\lambda-1-\mathrm{A})}{24 \beta}+\frac{\lambda-(1+\mathrm{A})}{12 \beta}+\frac{1}{12} \operatorname{Pr}^{2} \lambda \mathrm{A}(1+2 \mathrm{~A}) \\
-\frac{1}{24} \operatorname{Pr}^{2} \lambda(1+2 \mathrm{~A})+\frac{\operatorname{Pr}^{2} \lambda n_{1} n_{3}}{24}-\frac{n_{1} n_{2}}{24}
\end{array}\right) / 15120 \beta \quad \text { (17a-e) } \\
& T[2]=\frac{1}{2}(1+\mathrm{A}) \operatorname{Pr}, \\
& T[3]=\frac{1}{6}\left(n_{1}+2 \mathrm{~A} n_{3}\right) \operatorname{Pr}, \\
& T[4]=\frac{\operatorname{Pr} n_{1} n_{3}}{24}-\frac{1}{24} \operatorname{Pr}^{2}(1+2 \mathrm{~A})+\frac{1}{12} \operatorname{Pr}^{2} \mathrm{~A}(1+2 \mathrm{~A}), \\
& T[5]=\frac{\operatorname{Pr} n_{2}}{120}-\frac{1}{60} \operatorname{Pr}^{2}\left(n_{1}+2 \mathrm{~A} n_{3}\right)+\frac{1}{60} \operatorname{Pr}^{2} \mathrm{~A}\left(n_{1}+2 \mathrm{~A} n_{3}\right) \\
& T[6]=\frac{\operatorname{Pr} n_{2} n_{3}}{24}-\frac{1}{360} \operatorname{Pr}^{2} n_{1}\left(n_{1}+2 \mathrm{~A} n_{3}\right)-\frac{\operatorname{Pr}^{2} n_{1} n_{3}}{240}+\frac{1}{240} \operatorname{Pr}^{3}(1+2 \mathrm{~A})-\frac{1}{120} \operatorname{Pr}^{3} \mathrm{~A}(1+2 \mathrm{~A}) \\
& +\frac{\operatorname{Pr}^{2} \mathrm{~A} n_{1} n_{3}}{360}-\frac{1}{360} \operatorname{Pr}^{3} \mathrm{~A}(1+2 \mathrm{~A})+\frac{1}{360} \operatorname{Pr}^{3} \mathrm{~A}^{2}(1+2 \mathrm{~A})+\frac{\operatorname{Pr}(\lambda-(1+\mathrm{A}))}{720 \beta}, \\
& T[7]=\frac{\operatorname{Pr}^{2} n_{2}(1+2 \mathrm{~A})}{1008}-\frac{\operatorname{Pr}^{2} n_{1}{ }^{2} n_{3}}{1008}+\frac{\operatorname{Pr}^{3} n_{1}(1+2 \mathrm{~A})}{1008}-\frac{\operatorname{Pr}^{3} n_{1} \mathrm{~A}(1+2 \mathrm{~A})}{1008}-\frac{\operatorname{Pr}^{2} n_{2}}{1260}+\frac{1}{630} \operatorname{Pr}^{3}\left(n_{1}+2 \mathrm{~A} n_{3}\right) \\
& +\frac{1}{630} \operatorname{Pr}^{3} \mathrm{~A}\left(n_{1}+2 \mathrm{~A} n_{3}\right)+\frac{\operatorname{Pr}^{2} \mathrm{~A} n_{2}}{2520}-\frac{1}{12600} \operatorname{Pr}^{3}\left(n_{1}+2 \mathrm{~A} n_{3}\right)+\frac{1}{12600} \operatorname{Pr}^{3} \mathrm{~A}\left(n_{1}+2 \mathrm{~A} n_{3}\right) \\
& +\frac{\operatorname{Pr} n_{3}(\lambda-(1+\mathrm{A}))}{12600 \beta}+\frac{\lambda n_{3}+n_{2}-n_{1}(1+\mathrm{A})}{50400 \beta}
\end{aligned}
$$

The above procedure is continuous. By substituting Eqns. (17) and (18) in Eqn. (13) based on DTM, the closed form of the solutions can be written as: 


$$
\begin{aligned}
f(\eta)= & +\frac{n_{1} \eta^{2}}{2}+\frac{n_{2} \eta^{4}}{24}+\frac{(\lambda-1-\mathrm{A}) \eta^{5}}{120 \beta}+\frac{\left(\lambda n_{3}+n_{2}-n_{1}(1+\mathrm{A})\right) \eta^{6}}{720 \beta}+\frac{\left(\frac{\lambda-(1+\mathrm{A})}{2 \beta}+\frac{1}{2} \operatorname{Pr} \lambda(1+2 \mathrm{~A})-\frac{n_{1}{ }^{2}}{2}\right) \eta^{7}}{2520 \beta} \\
& +\frac{\left(\frac{\lambda n_{3}+n_{2}-n_{1}(1+\mathrm{A})}{6 \beta}+\frac{1}{6} \operatorname{Pr} \lambda\left(n_{1}+2 \mathrm{~A} n_{3}\right)+\frac{n_{2}}{6}-\frac{\mathrm{A} n_{2}}{6}\right) \eta^{8}}{6720 \beta}+\ldots \ldots \ldots \ldots \\
\theta(\eta)= & +n_{3} \eta+\frac{1}{2} \operatorname{Pr}(1+\mathrm{A}) \eta^{2}+\frac{1}{6} \operatorname{Pr}\left(n_{1}+2 \mathrm{~A} n_{3}\right) \eta^{3} \\
& +\left(\frac{\operatorname{Pr} n_{1} n_{3}}{24}-\frac{1}{24} \operatorname{Pr}^{2}(1+2 \mathrm{~A})+\frac{1}{12} \operatorname{Pr}^{2} \mathrm{~A}(1+2 \mathrm{~A})\right) \eta^{4} \\
& +\left(\frac{\operatorname{Pr} n_{2}}{120}-\frac{1}{60} \operatorname{Pr}^{2}\left(n_{1}+2 \mathrm{~A} n_{3}\right)+\frac{1}{60} \operatorname{Pr}^{2} \mathrm{~A}\left(n_{1}+2 \mathrm{~A} n_{3}\right)\right) \eta^{5}+\ldots \ldots \ldots \ldots . . . .
\end{aligned}
$$

From the above Eqns. (19) and (20) the unknown values $n_{1}, n_{2}, n_{3}$ can be calculated by utilizing boundary conditions mentioned in eqn. (9). Afterwards, substituting obtained values of $n_{1}, n_{2}, n_{3}$ into the Eqns. (19) and (20) for particular values of physical parameters $(A=0.5, \operatorname{Pr}=1.0, \lambda=1.0, \beta=$ 0.2 ), the expressions of $f(\eta)$ and $\theta(\eta)$ can be written as follows:

$$
\begin{aligned}
f(\eta)= & \eta-0.4304956992669373 \eta^{2}+0.04749727973586565 \eta^{4} \\
& -0.020833333333333332 \eta^{5}+0.006664688591255408 \eta^{6} \\
& -0.0012314545519338464 \eta^{7}+0.0003764688851289184 \eta^{8}+\ldots \ldots \ldots \\
\theta(\eta)= & 1-1.4717066543208086 \eta+1.0 \eta^{2}-0.3887830088091138 \eta^{3} \\
& +0.052796948772303454 \eta^{4}+0.02893860638762883 \eta^{5} \\
& -0.0195612055133296 \eta^{6}+0.005149209805037258 \eta^{7} \\
& -0.000410892698247331 \eta^{8}+\ldots \ldots \ldots \ldots
\end{aligned}
$$

In order to validate the present solution of the problem and confirm accuracy, the DTM solutions are compared with existing numerical results. Numerical solution of the problem is executed in the symbolic code, MATLAB. A good agreement between the present technique and numerical solution is achieved in Table 2 for the selected case $(A=0.5, \operatorname{Pr}=1.0, \lambda=1.0, \beta=0.2)$. Also, skin-friction values for Newtonian fluid and couple stress fluid are depicted in Table 3. Furthermore, the present DTM solutions are compared in Table 4 with the earlier Newtonian results $(\beta=0)$ of Ishak et al. [7] when the buoyancy term $\lambda \theta$ in Eqn. (7) is absent and $A=0$ (steady-state flow) with $\operatorname{Pr}=1.0$. Again, excellent correlation is attained which confirms the validity of the DTM computations. 
Table2: Comparison of the present results and numerical solution for $A=0.5, \operatorname{Pr}=1.0, \lambda=1.0, \beta=0.2$

\begin{tabular}{|lc|c|c|c|c|c|}
\hline $\boldsymbol{\eta}$ & & $\boldsymbol{f}(\boldsymbol{\eta})$ & & & $\boldsymbol{\theta}(\boldsymbol{\eta})$ & \\
& DTM & Numerical & Error & DTM & Numerical & Error \\
\hline 0 & 0.0 & 0.0 & 0.0 & 1.0 & 1.0 & 0.0 \\
\hline 0.1 & 0.09909 & 0.09954 & 0.00045 & 0.86149 & 0.86190 & 0.00040 \\
\hline 0.2 & 0.19639 & 0.19658 & 0.00018 & 0.74105 & 0.74161 & 0.00056 \\
\hline 0.3 & 0.28900 & 0.28924 & 0.00024 & 0.63672 & 0.63708 & 0.00036 \\
\hline 0.4 & 0.37604 & 0.37620 & 0.00014 & 0.54641 & 0.54657 & 0.00015 \\
\hline 0.5 & 0.45638 & 0.45650 & 0.00012 & 0.46785 & 0.46879 & 0.00093 \\
\hline 0.6 & 0.52891 & 0.52963 & 0.00071 & 0.40170 & 0.40183 & 0.00013 \\
\hline 0.7 & 0.59498 & 0.59531 & 0.00033 & 0.34093 & 0.34179 & 0.00086 \\
\hline 0.8 & 0.65288 & 0.65349 & 0.00060 & 0.29288 & 0.29373 & 0.00083 \\
\hline 0.9 & 0.70358 & 0.70426 & 0.00067 & 0.28933 & 0.28988 & 0.00085 \\
\hline 1.0 & 0.74698 & 0.74786 & 0.00087 & 0.24256 & 0.24555 & 0.00099 \\
\hline
\end{tabular}

Table 3: Values of $\frac{1}{2} C_{f} R e^{1 / 2}$ for viscous fluid $(\beta=0)$ and couple stress fluid $(\beta>0)$ for $\operatorname{Pr}=1.0$

\begin{tabular}{|c|c|c|c|}
\hline $\boldsymbol{\beta}$ & $\mathbf{A}$ & $\boldsymbol{\lambda}$ & $-\frac{\mathbf{1}}{\mathbf{2}} \boldsymbol{C}_{\boldsymbol{f}} \boldsymbol{R} \boldsymbol{e}^{\mathbf{1 / 2}}$ \\
\hline 0 & 0 & 0 & 1.042045 \\
\hline & & 1 & 0.633587 \\
\hline & 1 & 0 & 1.420100 \\
\hline 0.5 & & 1 & 1.347786 \\
\hline 1.0 & & & 1.507633 \\
\hline 1.5 & & & 1.654082 \\
\hline
\end{tabular}


Table 4: Values of $-\theta^{\prime}(0)$ for $\operatorname{Pr}=1.0$ and $\beta=0$.

\begin{tabular}{|c|l|c|c|cc|}
\hline A & $\lambda$ & Ishak et al. [7] & Numerical results & Present results & $\mathrm{n}=9$ \\
& & & & & $\mathrm{n}=10$ \\
\hline 0 & 0 & 1.0000 & 1.0581 & 1.0499 & 1.0579 \\
\hline & 1 & 1.0873 & 1.1090 & 1.1199 & 1.0006 \\
\hline & 2 & 1.1423 & 1.1514 & 1.1710 & 1.1411 \\
\hline & 3 & 1.1853 & 1.1880 & 1.2227 & 1.1735 \\
\hline 1 & 0 & 1.6820 & 1.6860 & 1.7096 & 1.6989 \\
\hline & 1 & 1.7029 & 1.7058 & 1.7396 & 1.7189 \\
\hline
\end{tabular}

\section{RESULTS AND DISCUSSION}

Flow and heat transfer characteristics for various fluid and geometric parameters have been computed and are presented graphically (Figs. 2 - 5). The influence of couple stress parameter $\beta$ on velocity $f^{\prime}(\eta)$ and temperature $\theta(\eta)$ profiles are shown in Fig. 2(a)-(b). Fig. 2(a) depicts the variation of $\beta$ on velocity. From this figure it is observed that an increase in $\beta$ values results in an upsurge in the velocity. This augmentation is due to presence of couple stresses which accelerate the flow and is consistent through the boundary layer transverse to the sheet. The momentum boundary layer thickness is increased substantially with couple stress. Also, it is observed that as $\beta$ become higher the thickness of the velocity boundary layer reduces.

Fig. 2(b) specifies the influence of $\beta$ on temperature profile. From this figure it is evident that a rise in $\beta$ values corresponds to increase in temperature and this behaviour is most pronounced at some distance from the sheet i.e. towards the free stream. The thermal boundary layer thickness is decreased for varying values of $\beta$. Although the couple stress effect does not arise in the thermal boundary layer Eqn. (8), via coupling with the momentum Eqn. (7) through the buoyancy term, $\lambda \theta$, and the convective terms in the energy Eqn. (8) i.e. $+f \theta^{\prime}-\theta f^{\prime}$, a strong effect is induced in the thermal field. The coupling of the two boundary layer equations vanishes for the forced convection case $(\lambda=0)$. Overall the weak couple stress fluid ( $\beta=0.5$ and this is closer to the Newtonian case) achieves lower velocity and temperatures than the strong couple stress fluid $(\beta>1)$. Couple stress effects therefore produce non-trivial effects in polymer heat transfer and this has also been confirmed by Stokes [45], Cowin [46] and Eringen [47] among others. From above two figures it is concluded that influence of $\beta$ on velocity profile is greater compared to that on the temperature profile. 
Figs. 3(a)-(b) depict the effect of parameter $A$ on velocity and temperature profiles through the boundary layer. The parameter, $A$, characterise the unsteadiness in the stretching sheet flow. Inspection of Fig. 3(a) reveals that velocity shows a decreasing trend for variation of unsteadiness parameter. As the parameter $A$ increases, the velocity decreases and reaches to zero along stretching sheet. This is due to fact that $A$ is inversely proportional to the stretching coefficient $a$. Thus, elevation in values of $A$ decreases the stretching ratio. As a result the velocity reduces. The variation of temperature profiles is shown in Fig. 3(b). It is noticed that influence of rising values of $A$ reduces the temperature field and augments the boundary layer thickness. In general it is noticed that effect of unsteadiness parameter $A$ on the temperature profile is more compared to velocity profile.

Figs. 4(a)-(b) respectively describes the influence of buoyancy ratio parameter $\lambda$ on velocity and temperature variables. From Fig. 4(a) it is seen that the influence of increasing values of $\lambda$ is to escalate the velocity. Physically $\lambda>0$ denotes assisting flow (heating of the fluid), $\lambda<0$ reveals opposing flow (cooling of the fluid) and $\lambda=0$ implies forced convection flow (absence of free convection currents). Also, as $\lambda$ augments results the increase in the temperature difference. This leads to an augmentation of the velocity and a corresponding reduction in momentum (hydrodynamic) boundary layer thickness. Also, as $\lambda$ augments results the increase in the temperature difference $\left(T_{w}-T_{\infty}\right)$. This leads to an augmentation of the velocity and a corresponding increase in momentum (hydrodynamic) boundary layer thickness.

The variation of convection paramater $\lambda$ on temperature profile is depicted in Fig. 4(b). Initially, as $\lambda$ increases, the temperature decreases drastically which leads to thinner boundary layer due domination of conduction. Also, an increasing $\lambda$ produces a marked decrease in temperatures i.e. the regime is heated significantly, and the thermal boundary layer thickness is lessened and this result in an increase in the magnitude of the wall temperature gradient. This in turn produces the upsurge in the wall heat transfer rate (see Table 3).

Figs. 5(a)-(b) illustrate the impact of Prandtl number Pr on flow-field profiles. Fig. 5(a) demonstrates the velocity profile. From this graph it is noticed that with greater values of Pr (lower thermal conductivity) the flow is decelerated, and the momentum boundary layer thickness is decreased. Similarly, temperature profiles are diminished with increasing Pr values as seen in Fig. 5(b). Also, the variation in the temperature profiles is more significant for small values of Pr when compared with higher values. It is remarked that the small values of $\operatorname{Pr}(<1)$ represent liquid-like materials, which have high thermal conductivity but low viscosity. However, high-viscosity oils are represented by large values of $\operatorname{Pr}(>1)$. For the case of Prandtl number of unity both momentum and thermal 
diffusion rates are equal. For $\operatorname{Pr}<1$ thermal diffusion rate exceeds momentum diffusion rate and vice versa for $\operatorname{Pr}>1$.

\section{CONCLUSIONS}

Motivated by simulating thermal non-Newtonian polymer stretching flows, a mathematical model has been developed for unsteady couple stress rheological boundary layer flow over a stretching sheet with heat transfer. Via an appropriate similarity transformation, the governing nonlinear timedependent partial differential boundary layer equations have been rendered into nonlinear ordinary differential equations with appropriate wall and free stream boundary conditions. The semi-analytical Differential Transform Method (DTM) has been employed to solve the reduced nonlinear coupled ordinary differential boundary value problem. A numerical solution is also obtained via the MATLAB built-in solver 'bvp4c' to validate the results. Further validation with published results from the literature is included. The present computations reveal that:

(i)The flow is accelerated (and hydrodynamic boundary layer thickness reduced) with increasing couple stress parameter whereas it is decelerated (and hydrodynamic boundary layer thickness increased) with unsteadiness parameter.

(ii) Temperature is elevated (and thermal boundary layer thickness enhanced) with couple stress parameter whereas temperature is depleted (and thermal boundary layer thickness reduced) with unsteadiness parameter.

(iii)The flow is accelerated with increasing positive buoyancy parameter (for heating of the fluid) whereas it is decelerated with increasing negative buoyancy parameter (cooling of the fluid).

(iv) Temperature and thermal boundary layer thickness are boosted with increasing positive values of buoyancy parameter.

(v) Increasing Prandtl number decelerates the flow (and increases hydrodynamic boundary layer thickness) whereas it reduces temperatures (and decreases thermal boundary layer thickness). 
(vi) Excellent accuracy is achieved with the DTM approach which holds significant potential for multi-physical thermal fluid dynamics modelling.

The current work has considered the Stokes couple stress model and has been confined to electrically non-conducting flow in the absence of electrical or magnetic fields. Future studies will examine Eringen micropolar non-Newtonian liquids [47] and consider electrohydrodynamic and magnetohydrodynamic heat transfer [48] for Sakiadis stretching sheet flows.

\section{Acknowledgements:}

The first author wishes to thank DST-INSPIRE (Code No. IF160028) for the grant of research fellowship and to Central University of Karnataka for providing the research facilities. The authors wish to express their gratitude to the reviewers who highlighted important areas for improvement in this article. Their suggestions have served to enhance the clarity and depth of the interpretation in particular.

\section{REFERENCES}

[1] B.C. Sakiadis, Boundary layer behavior on continuous solid surfaces: I boundary layer equations for two dimensional and axisymmetric flow, AIChE J. 7 (1961) 26-28.

[2] L.J. Crane, Flow past a stretching plate, Z. Angew. Math. Phy. (ZAMP) 21 (1970) 645-647.

[3] Chen C-H, Laminar mixed convection adjacent to vertical, continuously stretching sheets. Heat Mass Transf. 33:471-476 (1998).

[4] Mahapatra TR, Gupta AS., Heat transfer in stagnation-point flow towards a stretching sheet. Heat Mass Transf. 38:517-521(2002).

[5] Vajravelu K, Nayfeh J (1993) Convective heat transfer at a stretching sheet. Acta Mech 96:12271235 .

[6] Boutros YZ, Abd-el-Malek MB, Badran NA, Hassan HS., Lie-group method of solution for steady two-dimensional boundary-layer stagnation-point flow towards a heated stretching sheet placed in a porous medium. Meccanica 41:681-691(2006). 
[7] Ishak, R. Nazar, I. Pop, Boundary layer flow and heat transfer over an unsteady stretching vertical surface, Meccanica 44, 369-375(2009).

[8] Andersson HI, Aarseth JB, Dandapat BS (2000) Heat transfer in a liquid film on an unsteady stretching surface. Int J Heat Mass Transf 43:69-74.

[9] Na T.Y. and Pop I. (1996): Unsteady flow past a stretching sheet. Mech. Res. Comm., 23,.413422.

[10] Wang C.Y., Du G., Miklavi M. and Chang C.C. (1997): Impulsive stretching of a surface in a viscous fluid. SIAM J. Appl. Math., vol.57, pp.1-14.

[11] Elbashbeshy E.M.A. and Bazid M.A.A. (2004): Heat transfer over an unsteady stretching surface. - Heat Mass Transfer, vol.41, pp.1-4.

[12] Nazar R, Amin N., Filip D. and Pop I. (2004): Unsteady boundary layer flow in the region of the stagnation point on the stretching sheet. - Int. J. Eng. Sci., vol.42, pp.1241-1253.

[13] Devi CDS, Takhar HS, Nath G, Unsteady mixed convection flow in stagnation region adjacent to a vertical surface. Heat Mass Transf., 26, 71-79 (1991).

[14] K.R. Rajagopal, T.Y. Na, A.S. Gupta, Flow of a visco-elastic fluid over a stretching sheet, Rheol. Acta 23 (1984) 213-215.

[15] O. Anwar Bég, J. Zueco and S.K. Ghosh Unsteady natural convection of a short-memory viscoelastic fluid in a non-Darcian regime: network simulation, Chemical Engineering Communications, 198, 172-190 (2010).

[16] C-K. Chen, M-I. Char and J. W. Cleave Temperature Field in Non-Newtonian Flow over a Stretching Plate, Journal Mathematical Analysis Applications 151, 301-307 (1990).

[17] M. Subhas Abel, P.S. Datti and N. Mahesha "Flow and heat transfer in a power-law fluid over a stretching sheet with variable thermal conductivity and non-uniform heat source, International Journal of Heat and Mass Transfer 52, 2902-2913(2009).

[18] B. Sahoo Flow and heat transfer of a non-Newtonian fluid past a stretching sheet with partial slip, Commun Nonlinear Sci Numer Simulat. 15, 602-615 (2010). 
[19] A. Hamid, Hashim, M. Khan, A. Hafeez, Unsteady stagnation-point flow of Williamson fluid generated by stretching/shrinking sheet with Ohmic heating, International Journal of Heat and Mass Transfer 126, 933-940 (2018).

[20] V.K. Stokes, Couple stresses in fluids, Phy. Fluids, 9, 1709-15 (1966).

[21] O. Anwar Bég, S.K. Ghosh, S. Ahmed and T. A. Bég, Mathematical modelling of oscillatory magneto-convection of a couple stress biofluid in an inclined rotating channel, J. Mechanics Medicine and Biology, 12 (3) 1250050-1 to 1250050-35 (2012).

[22] J. V. Ramana Murthy, J. Srinivas and O. Anwar Bég, Entropy generation analysis of radiative heat transfer effects on channel flow of two immiscible couple stress fluids, J. Brazilian Soc. Mech Sci. Eng, 39, 2191-2202 (2017).

[23] D. Tripathi, A. Yadav and O. Anwar Bég, Electro-osmotic flow of couple stress fluids in a micro-channel propagated by peristalsis, European Physical Journal Plus, 132: 173-185 (2017).

[24] N. A. Khan, F. Riaz and N.A.Khan, Heat transfer analysis for couple stress fluid over a nonlinearly stretching sheet, Nonlinear Engineering, 2, 121-127 (2013).

[25] T. Hayat, M. Awais, A. Ambreen Safdar, and A.A. Hendi, Unsteady three dimensional flow of couple stress fluid over a stretching surface with chemical reaction. Nonlinear Analysis: Modelling and Control, 17, 47-59 (2012).

[26] T. Hayat, M. Mustafa, Z. Iqbal and A. Alsaedi, Stagnation-point flow of couple stress fluid with melting heat transfer Appl. Math. Mech. -Engl. Ed., 34(2), 167-176 (2013),

[27] U.S. Mahabaleshwar, I.E. Sarris, A.A. Hill, Giulio Lorenzini, Ioan Pop, An MHD couple stress fluid due to a perforated sheet undergoing linear stretching with heat transfer", International Journal of Heat and Mass Transfer 105 (2017) 157-167.

[28] M. Devakar, T. K.V. Iyengar, "Stokes' problems for an incompressible couple stress fluid", Nonlinear Analysis: Modelling and Control 1 (2), 181-190 (2008).

[29] Najeeb Alam Khan and Fatima Riaz, "Off centered stagnation point flow of a couple stress fluid towards a rotating disk", The Scientific World Journal (2014) Art. ID 163586.

[30] N. A. Khan, F. Riaz and F. Sultan, "Effects of chemical reaction and magnetic field on a couple stress fluid over a non-linearly stretching sheet", Eur. Phys. J. Plus (2014) 129: 18. 
[31] N. A. Khan, S. Aziz and N. A. Khan, "Numerical simulation for the unsteady MHD flow and heat transfer of couple stress fluid over a rotating disk", Plos One, 9(5) (2014) e95423.

[32] N. A. Khan and F. Riaz, "Effects of slip factors and couple stresses for stagnation point flow over a moving plate", Chinese Journal of Engineering, Volume2013, Article ID727826,13pages 2013, Article ID 727826, 1-13.

[33] J.K. Zhou, Differential Transformation and its Application for Electrical Circuits, Wuhan, Huazhong University Press, China, 1986.

[34] C.L. Chen, Y.C. Liu, Differential transformation technique for steady nonlinear heat conduction problems, Applied Mathematics and Computation 95 (1998) 155-164.

[35] O. Anwar Bég, M.M. Rashidi, A. Aziz and M. Keimanesh, Differential transform study of hypersonic laminar boundary layer flow and heat transfer over slender axisymmetric bodies of revolution, Int. J. Applied Mathematics and Mechanics, (6): 83 - 108 (2012).

[36] T. A. Bég, M.M. Rashidi, O. Anwar Bég and N. Rahimzadeh, Differential transform seminumerical simulation of biofluid-particle suspension flow and heat transfer in non-Darcian porous media, Computer Methods Biomechanics Biomedical Engineering, 16(8):896-907 (2013).

[37] O. Anwar Bég, M.M. Rashidi, and N. Freidooni Mehr, Second law analysis of hydromagnetic flow from a stretching rotating disk: DTM-Padé simulation of novel nuclear MHD propulsion systems, Frontiers of Aerospace Engineering, 2, 1, 29-38 (2013).

[38] D. Tripathi, O. Anwar Bég, P.K. Gupta, G. Radhakrishnamacharya and J. Mazumdar, DTM simulation of peristaltic viscoelastic biofluid flow in asymmetric porous media: a digestive transport model, Journal of Bionic Engineering 12 (4) 1-13 (2015).

[39] S. Sepasgozara,n, M. Farajib, P. Valipour, Application of differential transformation method (DTM) for heat and mass transfer in a porous channel, Propulsion and Power Research, 6(1):41-48 (2017).

[40] M. Hatami and D. Jing, Differential Transformation Method for Newtonian and non-Newtonian nanofluids flow analysis: Compared to numerical solution, Alexandria Engineering Journal (2016) $55,731-739$. 
[41] A. Mirzaaghaian, D.D. Ganji Application of differential transformation method in micropolar fluid flow and heat transfer through permeable walls, Alexandria Engineering Journal (2016) 55, 2183-2191.

[42] S. Mosayebidorcheh, Analytical investigation of the micropolar flow through a porous channel with changing walls, Journal of Molecular Liquids 196 (2014) 113-119.

[43] M. M. Rashidi, N. Rahimzadeh, M. Ferdows, Md. Jashim Uddin and O. Anwar Bég, Group theory and differential transform analysis of mixed convective heat and mass transfer from a horizontal surface with chemical reaction effects, Chemical Engineering Communications, 199 (2012) 1012-1043.

[44] M. M. Rashidi, A. Basiri Parsa, O. Anwar Bég, L. Shamekhi, S. M. Sadri and Tasveer A. Bég, Parametric analysis of entropy generation in magneto-hemodynamic flow in a semi-porous channel with OHAM and DTM, Applied Bionics and Biomechanics, 11, 47-60 (2014).

[45] V.K. Stokes, On some effects of couple stresses in fluids on heat transfer, ASME J. Heat Transfer, 91, 182-184 (1969).

[46] S.C. Cowin, The theory of polar fluids, Advances in Applied Mechanics, 14, 279-347 (1974).

[47] A.C. Eringen, Microcontinuum Field Theories: II- Fluent Media, Springer, New York (2001).

[48] D. Tripathi, R. Jhorar, O. Anwar Bég and A. Kadir, Electro-magneto-hydrodynamic peristaltic pumping of couple stress biofluids through a complex wavy micro-channel, J. Molecular Liquids, 236, 358-367 (2017). 


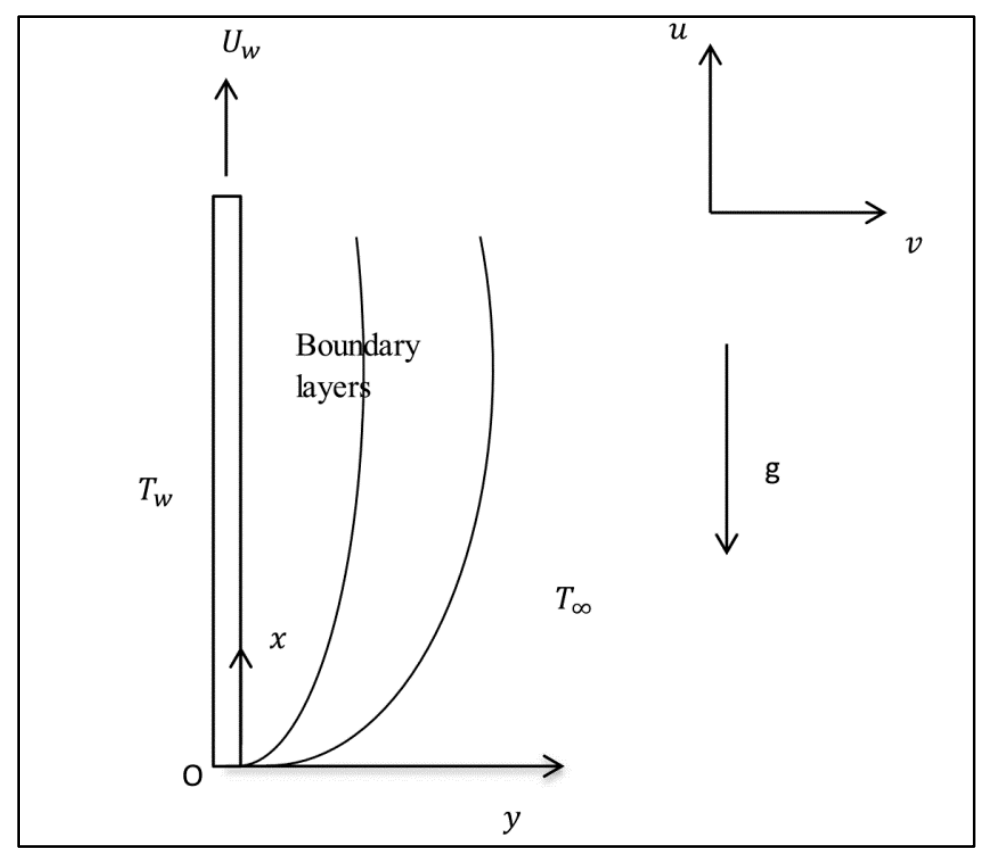

Fig. 1. Geometry of the present investigated problem 


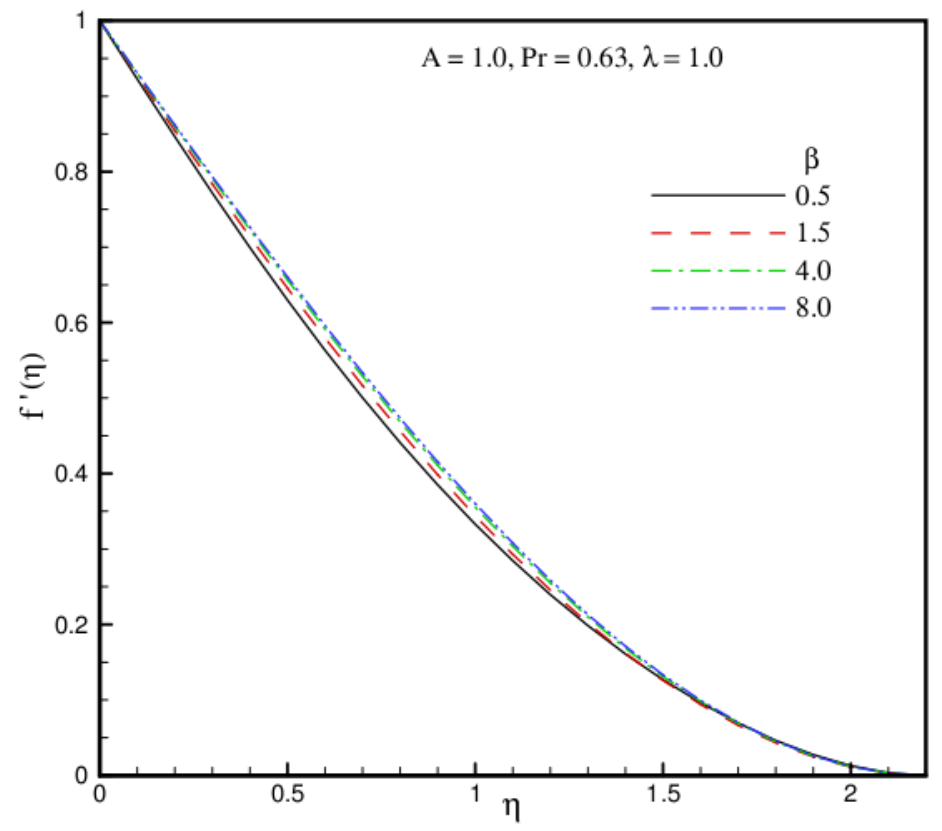

2(a) 


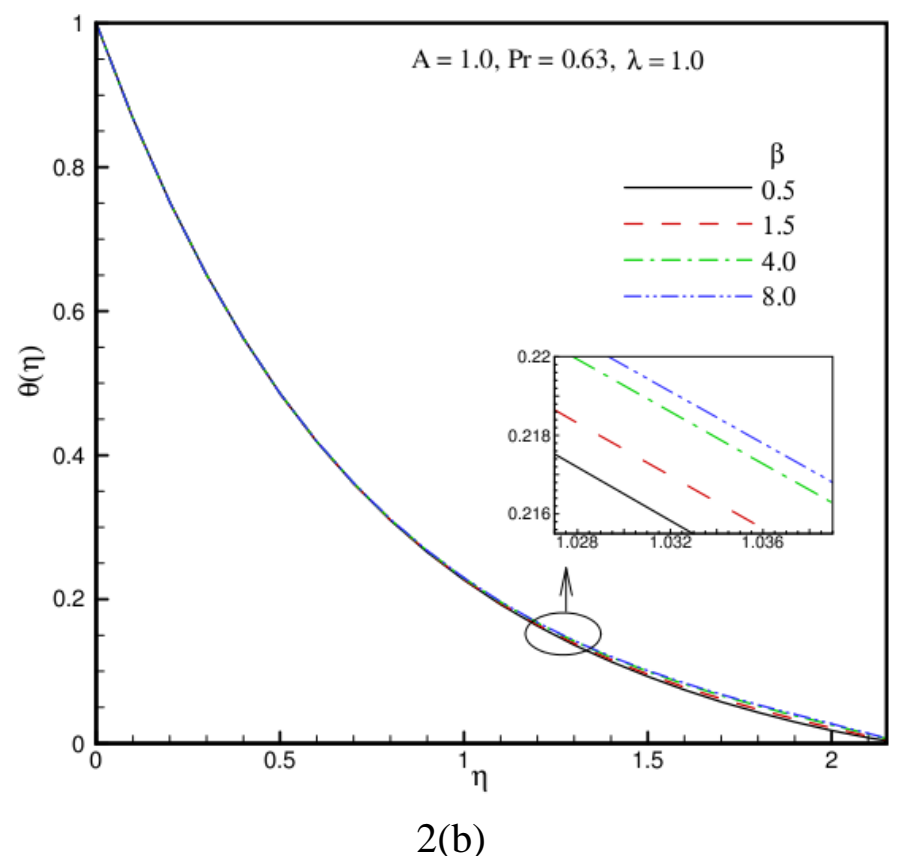

Fig. 2a, b. Simulated velocity and temperature profiles for various values of couple stress parameter.

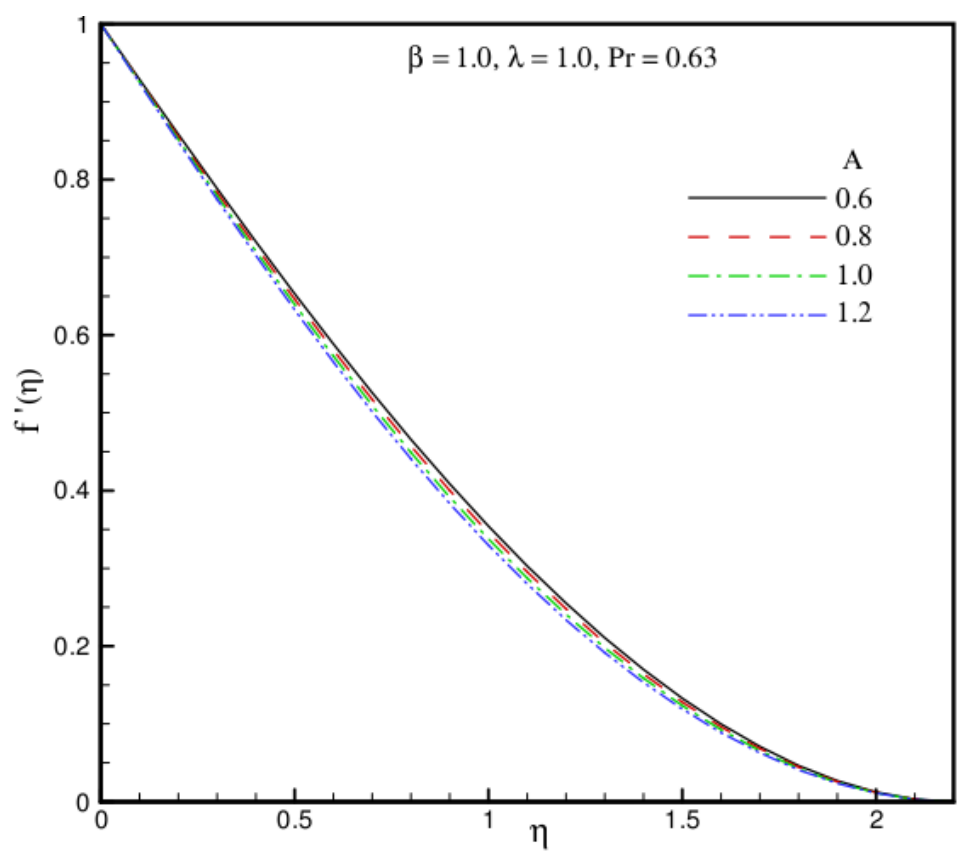


3(a)

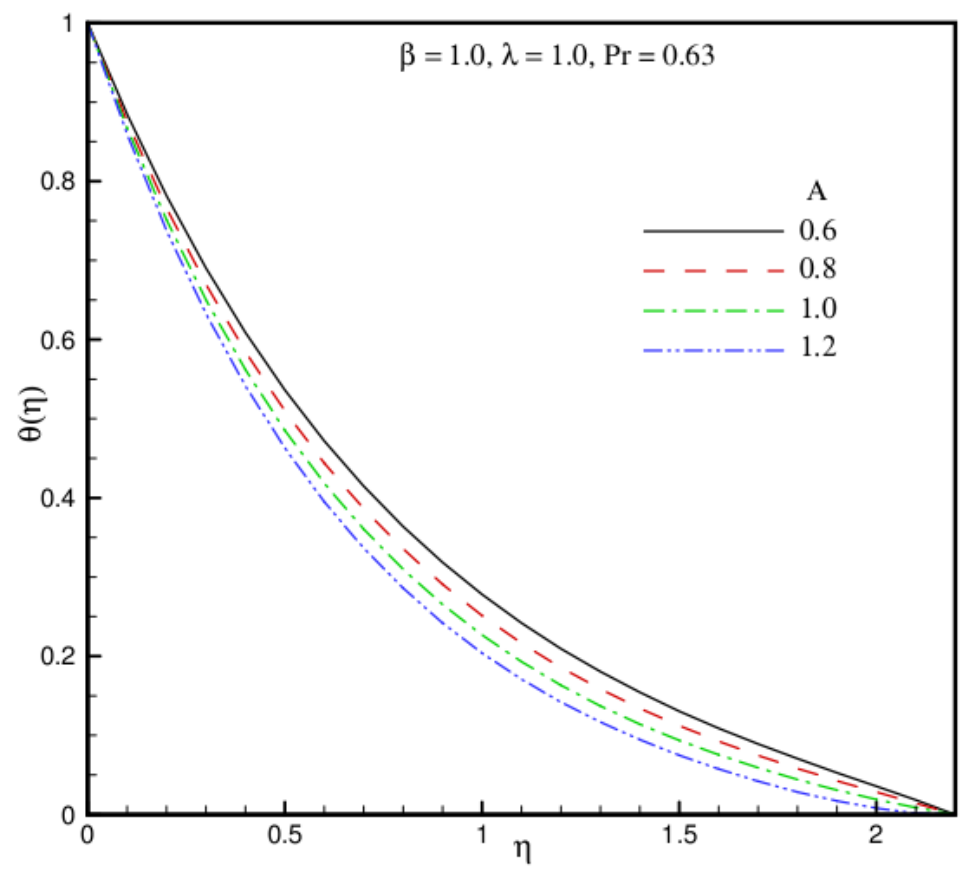

3(b)

Fig.3a,b. Simulated velocity and temperature profiles for different values of unsteadiness parameter.

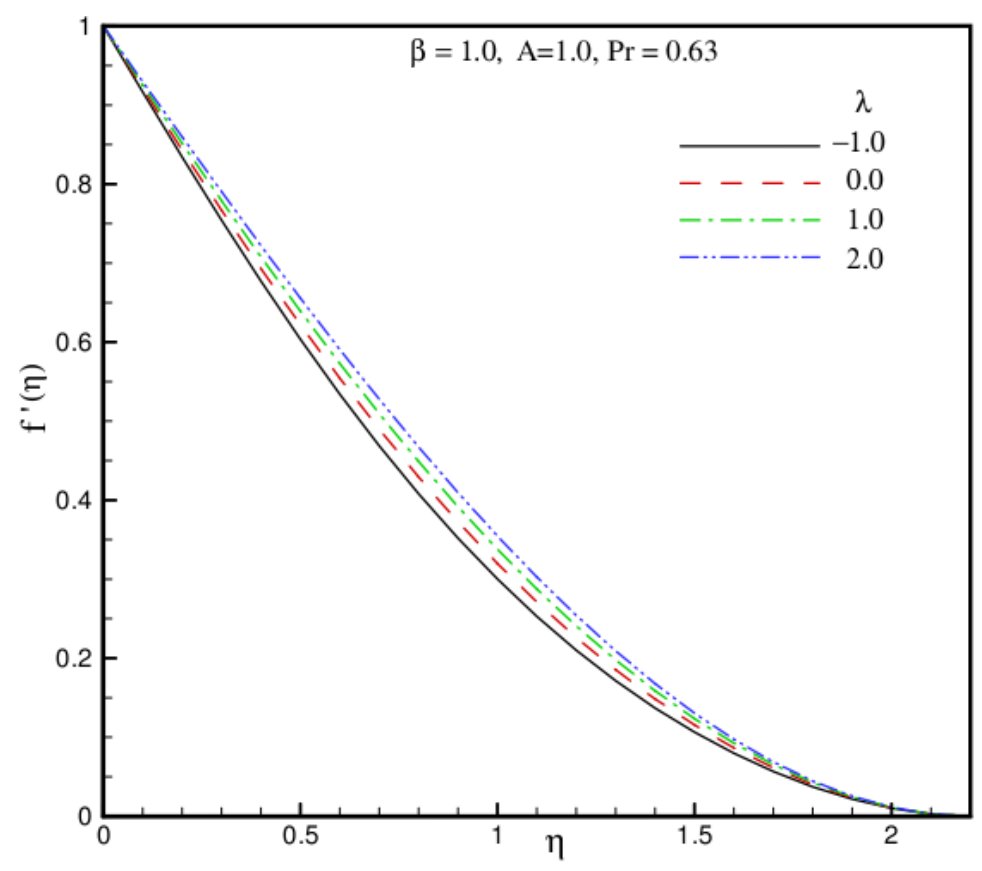

4(a) 


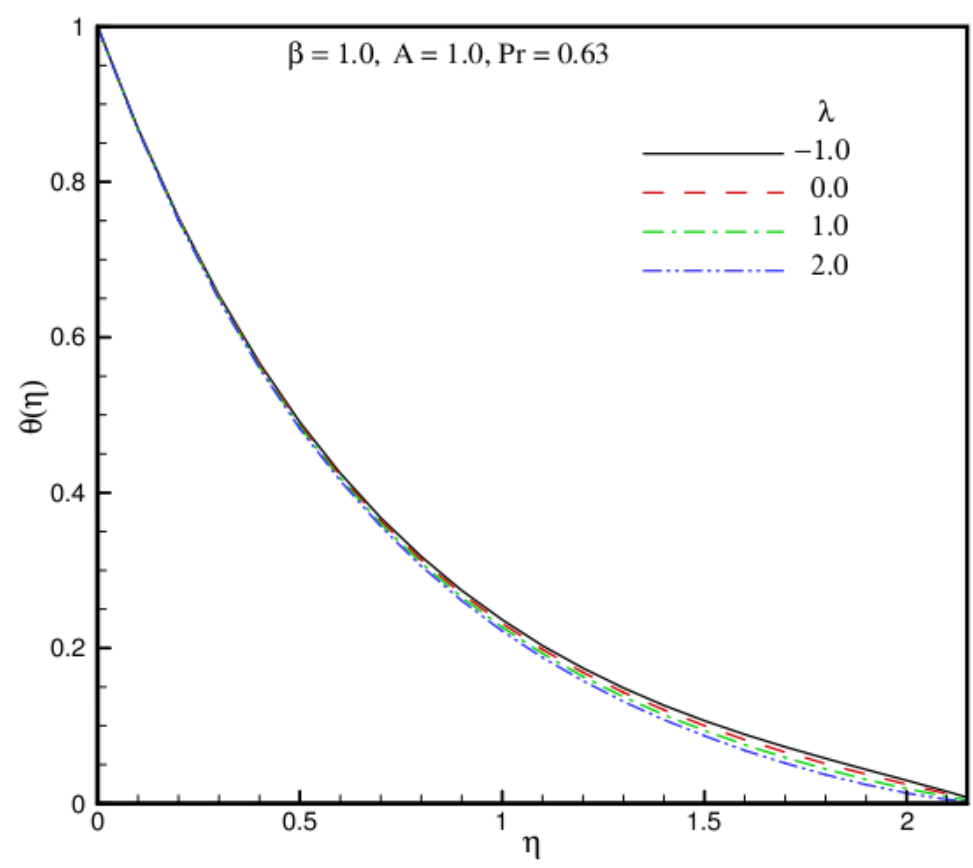

4(b)

Fig.4a,b. Simulated velocity and temperature graphs for various values of convection parameter.

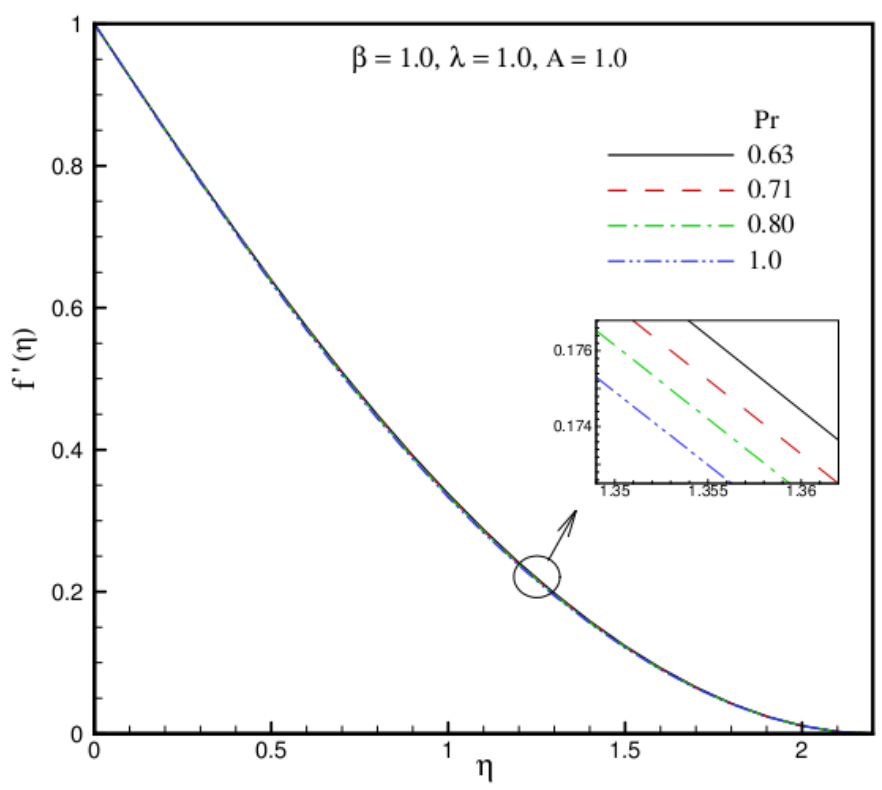

$5(a)$ 


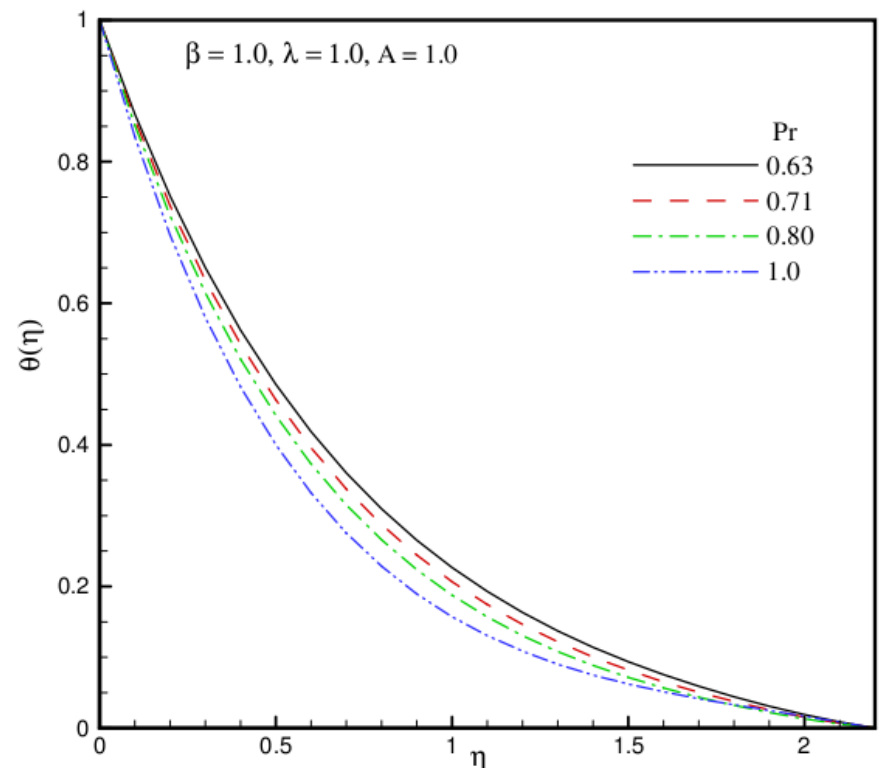

$5(b)$

Fig.5a, b. Simulated velocity and temperature profiles for various values of Prandtl number. 